\title{
BMJ Open Individually tailored whole-body vibration training to reduce symptoms of chemotherapy-induced peripheral neuropathy: study protocol of a randomised controlled trial - VANISH
}

\author{
Fiona Streckmann, ${ }^{\oplus 1,2,3}$ Viviane Hess, ${ }^{3}$ Wilhelm Bloch, ${ }^{2}$ Bernhard F Décard, ${ }^{4}$ \\ Ramona Ritzmann, ${ }^{5}$ Helmar C Lehmann, ${ }^{6}$ Maryam Balke, ${ }^{7}$ Christina Koliamitra, ${ }^{2}$ \\ Vanessa Oschwald, ${ }^{2}$ Thomas Elter, ${ }^{8}$ Lukas Zahner, ${ }^{1}$ Lars Donath, ${ }^{1,9}$ Ralf Roth, ${ }^{1}$ \\ Oliver Faude ${ }^{1}$
}

To cite: Streckmann F, Hess V, Bloch W, et al. Individually tailored whole-body vibration training to reduce symptoms of chemotherapyinduced peripheral neuropathy: study protocol of a randomised controlled trial-VANISH. BMJ Open 2019;9:e024467. doi:10.1136/ bmjopen-2018-024467

- Prepublication history for this paper is available online To view these files, please visit the journal online (http://dx.doi. org/10.1136/bmjopen-2018024467).

Received 31 May 2018 Revised 2 November 2018 Accepted 19 December 2018

Check for updates

(C) Author(s) (or their employer(s)) 2019. Re-use permitted under CC BY-NC. No commercial re-use. See rights and permissions. Published by BMJ.

For numbered affiliations see end of article.

Correspondence to Dr Fiona Streckmann; fiona.streckmann@unibas.ch

\section{ABSTRACT}

Introduction Chemotherapy-induced peripheral neuropathy (CIPN) is a prevalent and clinically meaningful side effect of cancer treatment. CIPN is induced by neurotoxic agents, causing severe sensory and/or motor deficits, resulting in disability and poor recovery, reducing patients' quality of life and limiting medical therapy. To date, effective treatment options are lacking. Whole-body vibration (WBV) training can attenuate motor and sensory deficits. We are conducting a two-armed, multicentre, assessor-blinded, randomised controlled trial, to investigate the effects of WBV on relevant symptoms of CIPN and determine the training characteristics.

Methods and analysis In this ongoing study, 44 patients who have completed therapy in the past 3 months, with a neurologically confirmed CIPN are assessed before and after a 12-week intervention and follow-up. The intervention group receives WBV twice a week. Exercises are individually tailored according to the initially determined optimal neuromuscular response. The control group receives care as usual. Primary endpoint is the patient reported reduction of CIPN-related symptoms (Functional Assessment of Cancer Therapy/Gynaecology Oncology Group-Neurotoxicity). Secondary endpoints are compound muscle action potentials, distal motor latency, conduction velocity, F-waves from the tibial and peroneal nerve, antidromic sensory nerve conduction studies of the sural nerve, normalised electromyographic activity, peripheral deep sensitivity, proprioception, balance, pain, the feasibility of training settings, quality of life and the level of physical activity.

Aim, ethics and dissemination The study was approved by both responsible ethics committees. (1) Our results may contribute to a better understanding of the effects of WBV on motor and sensory functions and (2) may provide information whether WBV at the most effective setting, is feasible for neuropathic patients. (3) Our results may also contribute to improve supportive care in oncology, thereby enhancing quality of life and enabling the optimal medical therapy. All results will be published in international peerreviewed journals as well as a manual for clinical practice.

\section{Strengths and limitations of this study}

With this study, we aim to detect the most effective (highest neuromuscular response), though feasible, setting for patients with chemotherapy-induced peripheral neuropathy (CIPN).

- The settings will then be evaluated directly in a 12week intervention.

- This is the first study to objectively evaluate the effects of different training settings of whole-body vibration on the symptoms of CIPN.

- We strive to create exercise recommendations that will be comprised in a manual and translated directly into practise.

- Due to the estimated sample size for the primary outcome, it is possible that the analysis of some secondary endpoints may be underpowered.

Trial registration number NCT03032718

\section{BACKGROUND}

Chemotherapy-induced peripheral neuropathy (CIPN) is the most common neurological and clinically relevant side effect of cancer treatment. Approximately $50 \%{ }^{1}$ of all patients with leukaemia, lymphoma, colorectal cancer or breast cancer are affected. ${ }^{2}$ CIPN can be induced by neurotoxic chemotherapeutic agents (platinum derivatives, vinca alkaloids, taxanes, as well as bortezomib, thalidomide and epothilones). ${ }^{1}$ Especially the peripheral nerves react very sensitive to these toxins, causing various sensory and motor dysfunctions. Patients suffer from symptoms such as reduced or absent Achilles tendon reflexes, loss of sensation such as numbness, tingling or burning, dysaesthesia, pain and loss of 
balance control leading to instable gait, as well as an increased incidence of accidents and falls. ${ }^{3-5}$ Patients experiencing these symptoms not only have to deal with the reduction in their autonomy and quality of life, but CIPN has become a crucial limiting factor for medical therapy, causing treatment delays, dose reductions or even discontinuation of therapy, which can affect the outcome and compromise survival. Consequently, the occurrence of CIPN presents a diagnostic dilemma because, up to now, approved and effective treatment options are lacking. ${ }^{12}$

Promising results have recently been revealed with specific exercise interventions. In a randomised controlled clinical trial, ${ }^{6}$ an exercise intervention consisting of endurance, strength and sensorimotor training twice a week for 36 weeks, accompanying lymphoma patients from diagnosis to completion of treatment, revealed a reduction of neuropathic symptoms. Patients in the intervention group showed a reduction of CIPN-related symptoms (eg, peripheral deep sensitivity) by $87 \%$, while no change $(0 \%)$ could be detected in the control group. After 36 weeks, $55 \%$ of the control group still had symptoms related to CIPN, while only $4 \%$ remained with CIPN in the intervention group. ${ }^{6}$

The majority of expertise on exercise and peripheral neuropathies arises from research on neuropathic diabetics. In a systematic review, ${ }^{7}$ exercise interventions for neuropathic patients independent of the derivation were evaluated. The results of this systematic review suggest that for toxically induced neuropathies such as CIPN, balance exercises were likely most beneficial in order to improve motor as well as sensory symptoms, though only if performed with sufficient intensity. Studies, for instance, that had only performed one or two balance exercises once a week showed no relevant effect. It therefore became apparent that the type of exercise is crucial and training parameters may also play a role as adaptations of the neuromuscular system are specific ${ }^{89}$ and depend on training volume and intensity. ${ }^{10}$

Targeting similar mechanisms as sensorimotor training, whole-body vibration (WBV) was also considered as a potential training modality. Previous studies in healthy adults, investigating WBV have shown a positive impact on parameters which can be affected by neuropathies. For instance, it has been shown that elderly improved their gait after vibration exercises, ${ }^{11}$ that WBV beneficially affected pain reduction ${ }^{12}$ and deconditioned skeletal muscle ${ }^{13}$ improved isometric strength, ${ }^{12}{ }^{14}$ postural sway ${ }^{15}$ and reduced fall frequency. ${ }^{16}$ Recently, a German group evaluated a nursing care program containing endurance, strength, functional training as well as vibration exercises (WBV) in 20 cancer patients. Patients reported less pain, better movement control and reduced discomfort from muscle atrophy and reduced loss of sensitivity. ${ }^{17} \mathrm{WBV}$ requires very little time and effort, but potentially has a high impact. For cancer patients, undergoing strenuous therapy, this can be an important factor. In addition, training and devices are feasible, meet the requirements of hospital hygiene and can be applied in all phases of therapy, even isolation. In a recent pilot study, cancer patients after treatment with neurologically confirmed CIPN were randomised to either WBV $(n=10)$, sensorimotor training $(\mathrm{n}=10)$ or a control group with no intervention $(n=10)$. WBV and sensorimotor training proved feasible for patients with CIPN. In addition, both exercise groups benefited (improved reflex activity, balance control and subjective reduction of symptoms) from 6 weeks of intervention twice a week. ${ }^{18}$ We consequently intend to further investigate the potentially beneficial effects of WBV in a randomized controlled trial (RCT). As seen in sensorimotor training (SMT), for instance, we know that the body reacts to the various settings differently so that it may be essential as to which setting is chosen-to date reporting on the precise setting is inconsistent or lacking. We therefore strive not only to evaluate the general effects of WBV but far more to determine the feasibility and effectiveness of the various settings. To our knowledge, no study has so far objectively and systematically evaluated the effects of WBV on the symptoms of CIPN. Furthermore, no indications exist regarding the feasibility of recommended settings for relevant symptoms of CIPN. For instance, studies investigating the effects of WBV to reduce pain, found that high frequencies $(>40 \mathrm{~Hz})$ were most beneficial. ${ }^{12}$ It remains unknown, however, whether neuropathic patients-especially suffering from neuropathic pain-would be able to tolerate such high frequencies. Exercise-induced neuromuscular activity is considered the most relevant factor affecting adaptations to various forms of sensorimotor or neuromuscular training. Ritzmann et $a l^{19}$ and Abercromby et $a l^{20}$ therefore, investigated variations in frequency, amplitude, position, load and type of the vibration platform with regard to the induced neuromuscular activity in order to define specific training recommendations for WBV in healthy, physically active adults. It is unclear, however, whether CIPN patients will respond similarly. Kneis et $a l^{5}{ }^{5}$ for instance, reported that the Ia afferent reflex pathway is affected in breast cancer patients, likely due to the sensory deficits caused by CIPN. Since WBV is considered to activate the muscle via spindle reflexes and motor unit recruitment and the discharge frequency, which are essential for the response, ${ }^{21}$ are influenced by the frequency and amplitude of WBV, training recommendations for patients may differ from healthy populations.

To summarise, WBV may hold potential for patients suffering from CIPN. It is therefore essential to gain a better understanding on the effects WBV can have on the symptoms of CIPN. In order to generate training recommendations and lay the foundation for future research, it is important to verify whether the settings potentially provoking the highest neuromuscular response are feasible and beneficial for patients with CIPN.

Based on the above-mentioned rationales, we aim to (a) investigate whether WBV can reduce relevant symptoms of CIPN, (b) investigate how CIPN patients adapt to an individually tailored WBV training regimen over a 12-week period, (c) investigate whether the training 
parameters provoking the highest neuromuscular response, are feasible for patients with CIPN, (d) detect an appropriate and feasible dose for training recommendations, particularly with regard to future clinical trials and (e) investigate the correlations with balance, the level of physical activity and patients' quality of life.

Based on previous pilot findings, we hypothesise that WBV will reduce relevant symptoms such as the loss of peripheral deep sensitivity, pain, weakened or absent reflexes or the loss of balance control. Additionally, we expect that these symptom reductions are accompanied by neurophysiological functional adaptations. We furthermore believe that WBV will be feasible for patients with CIPN, despite neuropathic pain, that patients will feel most comfortable within a certain range of training parameters that are lower than in healthy adults and that patients will experience an improvement in their level of physical activity and quality of life.

\section{METHODS/DESIGN}

\section{Patient and public involvement}

The study was designed without patient participation. All patients will recieve their individual results at the end of the study, as well as the final publication. We strive to find a treatment option for patients with CIPN in order to improve their autonomy, quality of life while still receiving the most suitable medical therapy. We therefore intend not only to publish the results in high-ranked, international journals but more importantly to create a manual (on paper as well as digital video and picture material) for patients, containing all training settings and exercises, as well as for therapists and physicians to distrubute to their patients.

\section{Study participants and recruitment}

In this ongoing study $(\mathrm{n}=8 / 44)$, oncological patients, aged 18-80 years, are recruited from two participating centres: The University Hospital Basel (Switzerland) and the University Hospital Cologne (Germany). Primary inclusion criteria are a performance status of 0-2 according to the toxicity and response criteria of the Eastern Cooperative Oncology Group as well as a completed chemotherapy in the past 3 months with one of the following neurotoxic agents: taxanes (docetaxel with a cumulative dose of $\geq 225 \mathrm{mg} / \mathrm{m}^{2}$ or paclitaxel with a cumulative dose of $\geq 525 \mathrm{mg} / \mathrm{m}^{2}$ ), vinca alkaloids (vincristine with a cumulative dose of $\geq 4.2 \mathrm{mg} / \mathrm{m}^{2}$ or vinblastine with a cumulative dose of $\geq 24 \mathrm{mg} / \mathrm{m}^{2}$ ), platinum derivatives (oxaliplatin with a cumulative dose of $\geq 510 \mathrm{mg} / \mathrm{m}^{2}$, cisplatinum with a cumulative dose of $\geq 200 \mathrm{mg} / \mathrm{m}^{2}$ ), in the past 3 months. Eligible patients are screened (with the Functional Assessment of Cancer Therapy/Gynaecology Oncology Group- Neurotoxicity [FACT/GOG-Ntx], ie, the primary endpoint) for neuropathic symptoms after chemotherapy. In case of a score $>0$, we will investigate further, inform the patient, consult the neurologist and perform neurophysiological tests to confirm a neuropathy (clinical diagnosis, nerve conduction velocity $<40 \mathrm{~m} / \mathrm{s}$ and an amplitude $<5 \mathrm{mV}$ and $\mu \mathrm{V}$, respectively), ensuring final eligibility. Exclusion criteria are a pre-existing neuropathy of other cause (eg, diabetes), given contraindications for WBV (instable osteolysis, osteosynthesis, acute thrombosis, foot ulcers and a fracture of a lower extremity in the last 2 years), a myocardial infarction, angina pectoris or heart disease (New York Heart Association [NYHA] IIIIV) within the past 6 months, a mental condition or lack of the German language that prevents the understanding of the written informed consent, metastases of the central nervous system and epilepsy. Relative exclusion criteria (inclusion or continuation after recovery possible) are an acute infection $\left(\geq 38.0^{\circ} \mathrm{C}\right.$ and / or intravenous therapy with antibiotics), thrombocyte count $<10 \times 10^{9} / \mathrm{L}$ and leucocyte count $<1 \times 10^{9} / \mathrm{L}$.

\section{Experimental design}

The study follows a prospective, multicentre, two-armed, randomised controlled design (table 1). Study participants are informed about the study procedure and are obliged to sign a written informed consent after a sufficient time for consideration. Patients are then assigned by an independent researcher, via a random generator, either to the intervention or the control group. Group assignment is revealed to the patient and the responsible coach after the first assessment. Patients in the intervention group receive a defined WBV exercise programme twice a week in addition to their usual treatment. Patients in the control group will receive treatment as usual and will be given the opportunity to participate in the intervention when the study is completed.

All patients are stratified to the intervention or control group (intervention:control=1:1) according to the minimisation method. ${ }^{22}$ Computerised minimisation is conducted by one researcher (OF) who has no direct contact to the patients and who is not directly involved in the intervention. The study centre, neurotoxic agent and age serve as strata for minimisation.

Data are assessed at three main timepoints and four short interim tests. The electrophysiological studies take place only once, prior to randomisation, in order to ensure the inclusion criteria. At baseline (T0), all assessments are performed. A short interim testing (T1) is performed, with the intention of detecting early signs of symptom reduction, as seen in pilot studies, after the first 6 weeks of training. Postsssessment (T2) takes place after 12 weeks. Patients are reassessed a further 12 weeks after the cessation of the intervention (T6), to evaluate the longterm effects of the intervention. During follow-up, a short status report is requested every 3 weeks (T3-T5) in order to enable timely and more precise feedback regarding the course of possible long-term effects (table 1). Each of the three main assessment will last $90 \mathrm{~min}$ at the most. Interim testing takes about $10 \mathrm{~min}$.

\section{Endpoints and assessment procedures}

In order to reduce bias, assessors will be blinded as to which arm patients are stratified and all assessments are 
Table 1 Standard Protocol Items: Recommendations for Interventional Trials flowchart-schedule of enrolment, interventions and assessments

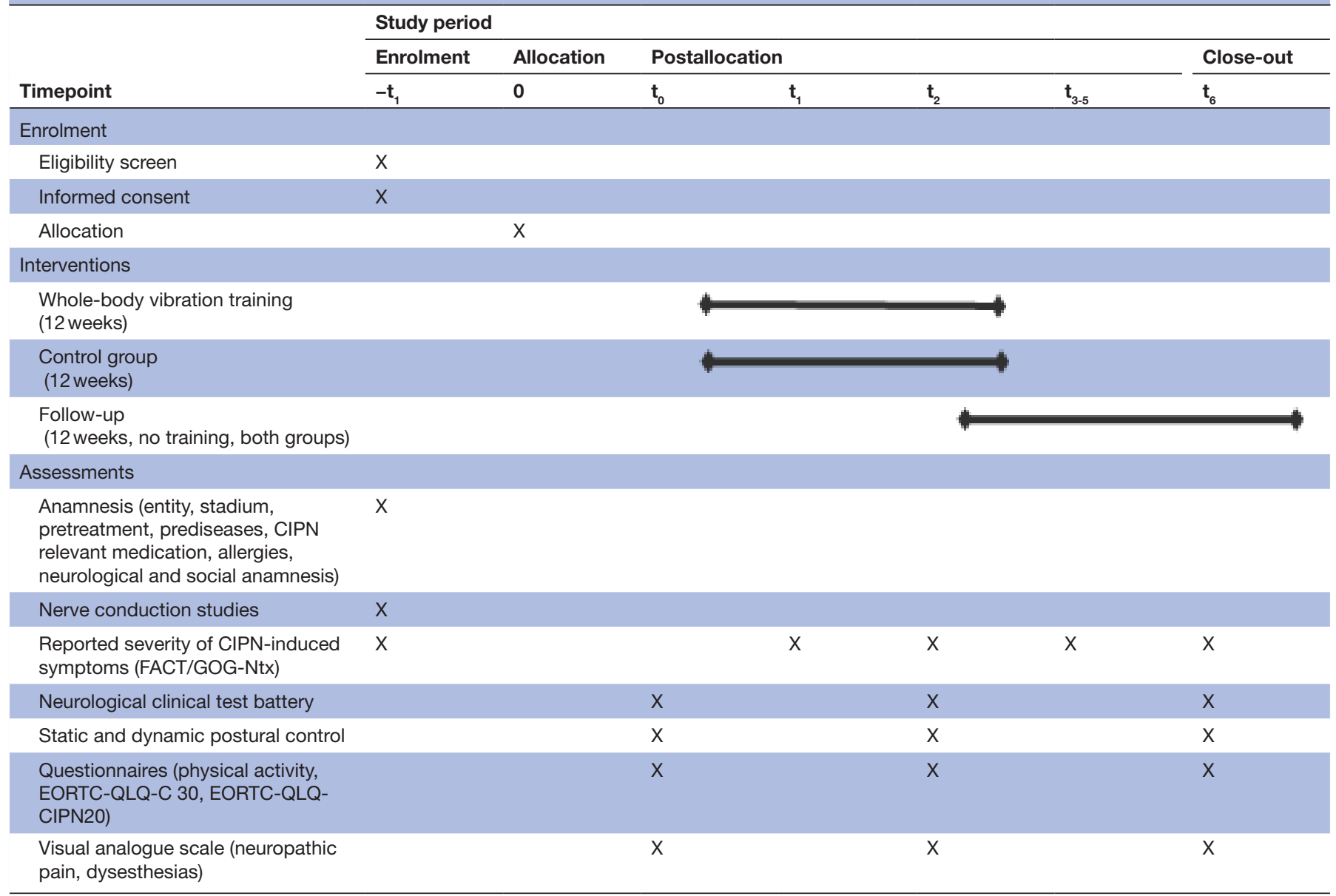

CIPN, chemotherapy-induced peripheral neuropathy; EORTC-QLQ-C, European Organization for Research and Treatment of Cancer Quality of Life Questionnaire; EORTC-QLQ-CIPN, European Organization for Research and Treatment of Cancer peripheral neuropathy questionnaire; FACT/GOGNtx, Functional Assessment of Cancer Therapy/Gynaecology Oncology Group-Neurotoxicity.

standardised. Both recruiting sites are equipped with the identical assessment tools. Assessments are performed at the Department of Sport, Exercise and Health of the University of Basel, as well as in the University Hospital Cologne. The same, trained and blinded investigator, at the same time of day, in the same room and maintaining a consistent temperature performs the tests within one individual. We have an expert for the assessments and an expert for the training, that will serve as 'gold-standard setters' for both sites. All assessors will be taught by these and have to undergo an exam as well as supervised test-runs prior to the study, using a standard operating procedure. Furthermore, patients are instructed not to exercise on testing days as well as the day before and not to reveal their group assignment to the investigators. In addition, testers have no contact with training staff in order to assure blinding during all assessments.

\section{Primary endpoint}

In accordance with pharmaceutical studies to reduce the symptoms of CIPN, ${ }^{23}$ we chose a patient reported outcome as primary endpoint: the FACT/GOG-Ntx questionnaire.
It is used to document and assess the severity of the subjective neuropathic symptoms. ${ }^{24}$ This questionnaire is reliable, has been validated (Cronbach $\alpha>0.7)^{25}$ and is widely applied in clinical practice. It contains 11 items which allow for an assessment of the extent of neuropathic symptoms-from 'not at all' to 'very much'. ${ }^{16}$

\section{Secondary endpoints}

In order to better understand the effects and underlying mechanisms as well as to reduce bias, we also assess the effects on the symptoms of CIPN by more objective, clinical measures. Since CIPN is associated with an entire symptom pattern, it is currently not possible to narrow it down to only one assessment method. We consequently conduct a comprehensive test battery, in order to target all relevant symptoms and signs of CIPN.

\section{Neurophysiological assessments}

Nerve conduction studies are performed prior to randomisation to objectively confirm CIPN. For nerve conduction studies, motor and sensory nerves will be assessed. Compound muscle action potentials, distal motor latency, 
conduction velocity and F-waves will be obtained from the tibial and peroneal nerve. The tibial nerve will be stimulated at the ankle and popliteal fossa. Antidromic sensory nerve conduction studies will be performed in the sural nerve. Sensory nerve action potentials will be recorded from the lateral malleolus with surface electrodes. Skin temperature will be monitored and maintained above $32^{\circ} \mathrm{C}$ using a heater if necessary.

We will furthermore conduct a standardised neurological clinical test battery, that is, valid and feasible in order to check for neuropathic symptoms. ${ }^{26}{ }^{27}$ Peripheral deep sensitivity is evaluated with a validated ${ }^{28}$ Rydel-Seiffer tuning fork $(128 \mathrm{~Hz})$ on a scale from 0 to 8 . Due to age-related neural deconditioning, values $\leq 4$ are pathological for patients older than 60 years, while for patients under 60 years old, $\leq 5$ is regarded as pathological. ${ }^{29}$ The reflex action of the Achilles tendon reflex as well as the patellar tendon reflex is assessed with a reflex hammer and graded on a three-point scale (1=agile, 2=weak, $3=$ =missing). Sense of position is examined by testing whether patients can recognise a change of position in their first toe, with their eyes closed. In order to assess perception of touch, the examiner symmetrically strokes the outsides of the patients' legs and feet in order to detect reduced or altered sensation due to demyelination or axonal degeneration. ${ }^{14} 30$ The strength of the leg muscles is assessed by requesting the patient to actively extend their legs against the resistance of the examiner's arm. The examiner then grades the strength on a six-point scale $(0=$ noactivity, $1=$ visual contraction without motor effect, $2=$ movement under elimination of gravity, $3=$ movement under gravity, $4=$ movement against slight resistance, $5=$ normal force).

\section{Neuropathic pain}

In line with pharmaceutical studies to reduce the symptoms of CIPN, we also use a visual analogue scale, starting at $0=$ no pain, up to $10=$ unbearable pain, ${ }^{23}$ in order to assess neuropathic pain as well as dysesthesia (any inappropriate sensation reported by the patient).

\section{Balance control}

A force plate (Leonardo, Novotec, Pforzheim, Germany) is used to assess changes in postural control as indicated by changes in the centre of pressure pathway length during upright static and dynamic stance. The assessment follows a standardised protocol (including various stands and eyes closed/open). Primarily, the supporting foot is determined with a short test. ${ }^{31}$ Patients are then asked to maintain an upright position with their knees slightly flexed $\left(\sim 30^{\circ}\right)$, hands at their side and their gaze straight ahead for $30 \mathrm{~s}$. The cumulative change in sway paths during this period is registered and serves as a measure of postural control. To minimise bias through potential learning effects, each position is repeated three times. Additionally, failed attempts are recorded. The exercises become progressively more difficult as previous studies (see Taube et $a \vec{l}^{22}$ for review) have shown that postural tasks with different complexity serve best to test for changes in stance stability after balance training. To assess a more 'dynamic' stance, a balance pad is additionally placed on top of the force plate.

We apply the EORTC-QLQ-C30 questionnaire to assess health-related quality of life. The questionnaire has been translated and validated ${ }^{33}$ into 81 languages and is used in $>3000$ studies worldwide. It is regarded reliable. $^{33} 34$

The Freiburg Physical Activity Questionnaire is a standardised and validated ${ }^{35}$ questionnaire that assesses the physical activities performed by a patient during the past 4 weeks. Based on the patients' answers, metabolic equivalent of task (MET) scores are calculated..$^{35} 36$

We apply the EORTC-QLQ-CIPN20, a phase IV questionnaire. ${ }^{37}$ It is a 20 -item questionnaire that was developed to elicit patients' experience of symptoms and functional limitations related to CIPN. ${ }^{38}$

Additionaly, patients will be asked to give feedback on a Borg-Scale after each setting, as well as 2 days later, regarding pain or any other discomfort. Should patients report any discomfort (eg, pain, muscle cramps) during the vibration setting, it will be aborted immediately and recorded as not feasible. Patients will also be monitored closely during each training and encouraged to report back any discomforts that may be related to the training.

\section{Procedures}

After a short familiarisation with the vibration plate, we strive to determine the setting that evokes the highest neuromuscular response in the individual patient of the intervention group, which will then be used as a guideline for all further training sessions. Patients are, therefore, exposed to 16 different conditions of WBV within the first two training sessions. These conditions vary in frequency $(10 \mathrm{~Hz} / 20 \mathrm{~Hz} / 30 \mathrm{~Hz} / 35 \mathrm{~Hz})$, amplitude $(2 \mathrm{~mm} / 4 \mathrm{~mm})$ and duration $(30 \mathrm{~s} / 60 \mathrm{~s})$. The order of the test conditions is randomised in order to control for confounding such as familiarisation or fatigue. Sets will be separated by a 5 min resting period normalised and integrated surface electromyography (EMG) of mm. tibialis anterior, soleus, gastrocnemius (medial head), mm. rectus femoris, vastus medialis, biceps femoris of the right leg is performed using bipolar $\mathrm{Ag} / \mathrm{AgCl}$ surface electrodes during the first two training sessions. A reference electrode is placed on the tibia. To keep interelectrode resistance $<2 \mathrm{k}$ and exclude vibration-induced artefacts, ${ }^{19}$ the skin areas for the electrodes are shaved, degreased and slightly abraded. The EMG signals will then be transmitted to the amplifier (band-pass filter $10 \mathrm{~Hz}-1 \mathrm{kHz}, 10009$ amplified) via shielded cables and recorded with $4 \mathrm{kHz}$. To detect the level of neuromuscular fatigue, the EMG traces for each of the recoded WBV conditions are divided into three equal parts regarding time (ie, $3 \times 20$ s). If the mean amplitude decreases by $>20 \%$ from one-third compared with the first part, ${ }^{39}$ patients are considered to be fatigued. Thus, we will choose the condition prior to this state as an appropriate WBV training stimulus. 


\section{Intervention}

Training is then based on the previously investigated individual setting that caused the highest neuromuscular response (between 10 and $35 \mathrm{~Hz}, 2-4 \mathrm{~mm}$ amplitude for 30 or $60 \mathrm{~s}$ ). Training sessions start directly after randomisation and are supervised by trained sport students. They take place twice a week, for 12 weeks in specific training rooms meeting the needs of oncological patients. The vibration exercises take place on a side-alternating vibration platform (Galileo, Pforzheim, Germany) according to the initially determined setting for each individual (highest, though feasible neuromuscular response). Patients are asked to stand barefoot to avoid dampening effects due to different footwear. Bodyweight should be on the forefoot without heel contact and knees are slightly bent. Patients should stand upright, looking straight ahead. The accurate position is monitored constantly and corrected if necessary by trained instructors. Each session lasts for about $15-30 \mathrm{~min}$ in total, leaving sufficient time for regeneration. Training consists of four vibration exercises, chosen from a standardised pool of exercises with increasing difficulty in order to allow for individual, optimal progression. All sessions and adherence are documented by the supervisor. Adverse events are documented and patients asked to give feedback regarding the feasibility and subjective impression of each individual setting. Patients are additionally asked about experiencing any possible adverse events following each intervention and at the beginning of each training. All training sessions will be supervised. With an emphasis on safety and monitoring procedures, each session will be protocolled, stance width and frequency will be individually adjusted and body position will be controlled. Individual perception of the level of difficulty will be established immediately after each setting, with the validated and reliable Borg scale. ${ }^{40}$

\section{Statistical procedures and sample size estimation}

As primary analysis we will calculate two-factorial repeated measures analyses of variances (rANOVA; factor 1: control vs intervention group; factor 2: timepoint of measurement; pre, post, follow-up) to assess the time course of adaptations between groups. The main effect of interest is the rANOVA interaction term between both factors (Hecksteden et al., Front Physiol 2018). ${ }^{41}$ We use the exact $p$ value as a measure of the strength of evidence against the null hypothesis, that is, that there is no intervention effect (Sterne and Davey Smith, BMJ 2001). ${ }^{42}$ In order to arrive at an estimation of the size of the observed effects, we calculate the change scores from pre to post as well as pre to follow-up tests together with $90 \%$ CIs (Sterne and Davey Smith, BMJ 2001). ${ }^{42}$ We will perform intended-to-treat analyses. In case of missing data, we will conduct multiple imputation (k-nearest neighbour method). Based on pilot results, a medium effect for the primary endpoint can be expected. In this randomised, controlled pilot study, ${ }^{18}$ the FACT/GOG-Ntx sum score changed by $24 \%(16.8 \pm 8.1$ to $12.8 \pm 7.8)$ after 6 weeks of
WBV training in 10 oncological patients, whereas the control group remained similar. Based on this pilot study, medium effect sizes seem also a realistic and relevant estimate for several of the secondary outcomes (eg, postural balance, pain). As our training period is twice as long as in the pilot study, a medium effect size seems to be a realistic and conservative estimate. Assuming a medium effect $(\mathrm{f}=0.25)$ for the analyses of variances group $\mathrm{x}$ time interaction term (statistical power $90 \%$, two-sided significance level $\alpha=0.05$ ), 36 volunteers (18 per group) are needed for statistical analyses. We expect a realistic drop-out rate of about $20 \%$. Thus, a total of 44 patients are initially recruited. We will also conduct a sensitivity analysis by comparing the imputed results with the complete-case results.

Overall data management and data analyses will be conducted by three researchers (OF, LD and FS). Data entry will be double-checked and data ranges will be controlled for plausibility.

\section{Ethics and dissemination}

All results will be published in international peer-reviewed journals. We strive to create recommendations in a manual for clinical practice.

\section{DISCUSSION}

\section{Expected key results}

To date, there is no effective treatment option to deal with neuropathies though it presents a diagnostic dilemma as physicians need to find the balance between patients' quality of life and the effectiveness of the medical therapy. Our primary endpoint is therefore to evaluate the potential of WBV training to reduce the symptoms of CIPN and to find out the most appropriate and feasible training characteristics for neuropathic cancer patients. We expect that WBV will reduce the severity of the subjective CIPN-related symptoms as well as further relevant symptoms and signs such as the loss of peripheral deep sensitivity, pain, weakened or absent reflexes or the loss of balance control. Furthermore, it seems likely that WBV will be feasible for patients with CIPN, despite neuropathic pain, that patients will feel most comfortable within a certain, individual range of training parameters that are lower than in healthy adults and that patients will experience an improvement in their quality of life.

\section{Benefits and risks}

There is a chance patients will be able to reduce their debilitating symptoms of the CIPN without any further side effects. We do not expect any complications. The intervention has no negative influence on their therapy. Both groups will be given the optimal medical standard. However, we have to account for the possibility that patients with neuropathic pain in the lower extremities may possibly experience some pain during the vibration exercises at higher frequencies. Due to the low submaximal intensity, the position taken on the platform, as well 
as the well-established, non-invasive assessment methods, we estimate the potential risk for patients as very low. The electroneurography is a neurological routine assessment, that is, not associated with any specific risk. Due to the fact that electricity is used, it is possible that some patients may experience this sensation as uncomfortable or painful. ${ }^{38}$ Regarding the EMG assessment, there are also no known associated risks. It is, however, possible that the disinfecting and slight abrasion of the skin, that is, necessary to attach the surface electrodes (according to the international surface EMG for non-invasive assessment of muscles guidelines) can lead to reddening of the skin in that area right after the assessment.

\section{Potential for bias}

In order to minimise bias, we take several measures: patient allocation using the minimisation procedure according to Pocock and Simon ${ }^{22}$ allows for a balanced allocation of patients to both groups so that potential baseline differences in relevant confounders (age, study centre, neurotoxic agent) are minimised. Due to the fact that within this study setting, it is essential to supervise patients during training sessions by qualified exercise therapists, patients will subsequently know which arm they are in. Therefore, investigators performing the assessments are blinded as to which arm patients are in and are not allowed to train the patients and vice versa. All assessments are standardised as well as aligned among the investigators by gold-standard setters. In addition, patients will be requested to refrain from revealing the result of randomisation to any investigator except of course the exercise therapist. The study can therefore be considered an assessor-blinded study. The order of the test conditions will be randomised with the intention of controlling for confounding such as familiarisation or fatigue. To further reduce bias, both recruiting sites are equipped with the identical assessment tools. The study coordinator (FS) is the same for both study centres and training of study assistants is identical. All assessments within one individual will always be performed by the same trained investigator. Assessments are performed according to standardised operating procedures, at the same time of day, in the same room and maintaining a consistent temperature. ${ }^{38}$

\section{Perspectives}

Positive results will contribute to improve supportive care in oncology, consequently enhancing quality of life, enabling the optimal medical therapy in neuropathic cancer patients and, eventually, possibly even impacting survival for these patients. Thus, patients' social reintegration may be improved. Our results might reveal reference values with regard to the neuromuscular capabilities of CIPN patients, lead to a better understanding of the effects of WBV on motor and sensory functions and will provide information whether $\mathrm{WBV}$ is feasible for patients suffering from CIPN. We aim at publishing the results in peer-review scientific journals raising the awareness of the scientific community for this topic. Furthermore, we will create guidelines, training recommendations and manuals for clinical practice and healthcare professionals that can directly be translated patients' everyday life and can be performed on other vibration plates in a gym, hospital or physiotherapy practice, for instance. Finally, our results will form the foundation for future research on this topic.

\section{Author affiliations}

${ }^{1}$ Department of Sport, Exercise and Health, University of Basel, Basel, Switzerland ${ }^{2}$ Institute of Cardiovascular Research and Sport Medicine, German Sport University, Cologne, Germany

${ }^{3}$ Department of Oncology, University Hospital, Basel, Switzerland

${ }^{4}$ Department of Neurology, University Hospital, Basel, Switzerland

${ }^{5}$ Department of Sport and Sport Science, University of Freiburg, Freiburg i.Br.,

Germany

${ }^{6}$ Department of Neurology, University Hospital, Cologne, Germany

${ }^{7}$ Neurological Day Clinic (NTC) and Department of Early Neurological and

Interdisciplinary Rehabilitation, St. Marien-Hospital, Cologne, Germany

${ }^{8}$ Department of Oncology, University Hospital, Cologne, Germany

${ }^{9}$ Department of Intervention Research in Exercise Training, German Sports University, Cologne, Germany

Contributors FS and OF designed the study protocol. VH, BFD, RRi, WB, HCL and TE contributed to the design of the study. FS organises the study in both countries (ie, recruitment, data collection, quality control). FS, VH and TE are responsible for patient recruitment. BFD, HCL and MB are responsible for neurological assessments. RRi, RRo and LD support electromyographic assessment, analysis and interpretation. CK is responsible for the electromyographic assessment and training and VO for patient coordination and organisation in Cologne. FS, LZ, RRo, $\mathrm{LD}$ and $\mathrm{OF}$ designed the exercise intervention programme. FS, $\mathrm{LD}$ and $\mathrm{OF}$ conduct the overall data management and organise data preparation. OF and LD analyse the data. FS and OF wrote the present manuscript. All authors revised the study protocol, read and approved the final manuscript. FS is the guarantor.

Funding This study is funded by the 'Research Fund for Junior Researchers' of the University of Basel (DMS2345). FS is partly funded by a grant (grant number 70112048) of the German Cancer Aid (Deutsche Krebshilfe-DKH). The study funders have no influence on study design, collection, management, analysis and interpretation of data, writing of the report and the decision to submit the report for publication. Proofs of ethics approval and funding by email to the editorial office.

Competing interests None declared.

Patient consent for publication Not required.

Ethics approval The study was approved by both responsible ethics committees (Basel, Switzerland/Cologne, Germany).

Provenance and peer review Not commissioned; externally peer reviewed.

Open access This is an open access article distributed in accordance with the Creative Commons Attribution Non Commercial (CC BY-NC 4.0) license, which permits others to distribute, remix, adapt, build upon this work non-commercially, and license their derivative works on different terms, provided the original work is properly cited, appropriate credit is given, any changes made indicated, and the use is non-commercial. See: http://creativecommons.org/licenses/by-nc/4.0/.

\section{REFERENCES}

1. Wonders KY, Reigle BS, Drury DG. Treatment strategies for chemotherapy-induced peripheral neuropathy: potential role of exercise. Oncol Rev 2010;4:117-25.

2. Stubblefield MD, Burstein HJ, Burton AW, et al. NCCN Task force report: management of neuropathy in cancer. Journal of the National Comprehensive Cancer Network 2009;7:S-1-0.

3. Pietrangeli A, Leandri M, Terzoli E, et al. Persistence of high-dose oxaliplatin-induced neuropathy at long-term follow-up. Eur Neurol 2006;56:13-16.

4. Lehky TJ, Leonard GD, Wilson $\mathrm{RH}$, et al. Oxaliplatin-induced neurotoxicity: acute hyperexcitability and chronic neuropathy. Muscle Nerve 2004;29:387-92. 
5. Kneis S, Wehrle A, Freyler K, et al. Balance impairments and neuromuscular changes in breast cancer patients with chemotherapy-induced peripheral neuropathy. Clin Neurophysiol 2016;127:1481-90.

6. Streckmann F, Kneis S, Leifert JA, et al. Exercise program improves therapy-related side-effects and quality of life in lymphoma patients undergoing therapy. Ann Oncol 2014;25:493-9.

7. Streckmann F, Zopf EM, Lehmann HC, et al. Exercise intervention studies in patients with peripheral neuropathy: a systematic review. Sports Med 2014;44:1289-304.

8. Giboin LS, Gruber M, Kramer A. Task-specificity of balance training. Hum Mov Sci 2015;44:22-31.

9. Donath L, Roth R, Zahner L, et al. Slackline Training (Balancing over narrow nylon Ribbons) and balance performance: a meta-analytical review. Sports Med 2017;47:1075-86.

10. Lesinski M, Hortobágyi T, Muehlbauer T, et al. Dose-response relationships of balance training in healthy young adults: a systematic review and meta-analysis. Sports Med 2015;45:557-76.

11. Kawanabe K, Kawashima A, Sashimoto I, et al. Effect of whole-body vibration exercise and muscle strengthening, balance, and walking exercises on walking ability in the elderly. Keio J Med 2007;56:28-33

12. Rittweger J. Vibration as an exercise modality: how it may work, and what its potential might be. Eur J Appl Physiol 2010;108:877-904.

13. Blottner D, Salanova M, Püttmann B, et al. Human skeletal muscle structure and function preserved by vibration muscle exercise following 55 days of bed rest. Eur J Appl Physiol 2006;97:261-71.

14. Cochrane DJ. Vibration exercise: the potential benefits. Int J Sports Med 2011;32:75-99.

15. Spiliopoulou SI, Amiridis IG, Tsigganos G, et al. Vibration effects on static balance and strength. Int J Sports Med 2010;31:610-6.

16. Bogaerts A, Delecluse C, Boonen S, et al. Changes in balance, functional performance and fall risk following whole body vibration training and vitamin D supplementation in institutionalized elderly women. A 6 month randomized controlled trial. Gait Posture 2011;33:466-72.

17. Kirchner E. Pflegerische Interventionen und Möglichkeiten bei krebstherapiebedingter Polyneuropathie. DLH-INFO 2008;37:19-21.

18. Streckmann F, Lehmann HC, Balke M, et al. Sensorimotor training and whole-body vibration training have the potential to reduce motor and sensory symptoms of chemotherapy-induced periphera neuropathy-a randomized controlled pilot trial. Support Care Cancer 2018.

19. Ritzmann R, Kramer A, Gruber M, et al. EMG activity during whole body vibration: motion artifacts or stretch reflexes? Eur J Appl Physiol 2010;110:143-51.

20. Abercromby AF, Amonette WE, Layne CS, et al. Vibration exposure and biodynamic responses during whole-body vibration training. Med Sci Sports Exerc 2007;39:1794-800.

21. Gollhofer A, Horstmann GA, Berger W, et al. Compensation of translational and rotational perturbations in human posture: stabilization of the centre of gravity. Neurosci Lett 1989;105:73-8.

22. Pocock SJ, Simon R. Sequential treatment assignment with balancing for prognostic factors in the controlled clinical trial. Biometrics 1975;31:103-15.

23. Bennett MI, Laird B, van Litsenburg C, et al. Pregabalin for the management of neuropathic pain in adults with cancer: a systematic review of the literature. Pain Med 2013;14:1681-8.
24. Hayes S, Newman B. Exercise in cancer recovery: an overview of the evidence. Cancer Forum 2006;30.

25. Calhoun EA, Welshman EE, Chang $\mathrm{CH}$, et al. Psychometric evaluation of the Functional Assessment of Cancer Therapy/ Gynecologic Oncology Group-Neurotoxicity (Fact/GOG-Ntx) questionnaire for patients receiving systemic chemotherapy. Int $J$ Gynecol Cancer 2003;13:741-8.

26. Chaudhry V, Chaudhry M, Crawford TO, et al. Toxic neuropathy in patients with pre-existing neuropathy. Neurology 2003;60:337-40.

27. Cavaletti G, Cornblath DR, Merkies IS, et al. The chemotherapyinduced peripheral neuropathy outcome measures standardization study: from consensus to the first validity and reliability findings. Ann Oncol 2013;24:454-62.

28. Kästenbauer T, Sauseng $\mathrm{S}$, Brath $\mathrm{H}$, et al. The value of the RydelSeiffer tuning fork as a predictor of diabetic polyneuropathy compared with a neurothesiometer. Diabet Med 2004;21:563-7.

29. Pataky Z, Herrmann FR, Regat D, et al. The at-risk foot concerns not only patients with diabetes mellitus. Gerontology 2008;54:349-53.

30. White CM, Pritchard J, Turner-Stokes L. Exercise for people with peripheral neuropathy (Review). John Wiley \& Sons, Ltd: The Cochrane Collaboration, 2010.

31. Coren S. The lateral preference inventory for measurement of handedness, footedness, eyedness, and earedness: Norms for young adults. Bull Psychon Soc 1993;31:1-3.

32. Taube W, Gruber M, Gollhofer A. Spinal and supraspinal adaptations associated with balance training and their functional relevance. Acta Physiol 2008;193:101-16.

33. Aaronson NK, Ahmedzai S, Bergman B, et al. The European Organization for Research and Treatment of Cancer QLQ-C30: a quality-of-life instrument for use in international clinical trials in oncology. J Natl Cancer Inst 1993;85:365-76.

34. Emery CF. Cognitive functioning among patients in cardiopulmonary rehabilitation. J Cardiopulm Rehabil 1997;17:407-10.

35. Frey I, Berg A, Grathwohl D, et al. [Freiburg Questionnaire of physical activity-development, evaluation and application]. Soz Praventivmed 1999;44:55-64.

36. Ainsworth BE, Haskell WL, Herrmann SD, et al. 2011 Compendium of physical activities: a second update of codes and MET values. Med Sci Sports Exerc 2011;43:1575-81.

37. Postma TJ, Aaronson NK, Heimans JJ, et al. The development of an EORTC quality of life questionnaire to assess chemotherapyinduced peripheral neuropathy: the QLQ-CIPN20. Eur J Cancer 2005;41:1135-9.

38. Streckmann F, Balke M, Lehmann HC, et al. The preventive effect of sensorimotor- and vibration exercises on the onset of Oxaliplatin- or vinca-alkaloid induced peripheral neuropathies - STOP. BMC Cancer 2018;18:62.

39. Gandevia SC. Spinal and supraspinal factors in human muscle fatigue. Physiol Rev 2001;81:1725-89.

40. Cleland BT, Ingraham BA, Pitluck MC, et al. Reliability and validity of ratings of perceived exertion in persons with multiple sclerosis. Arch Phys Med Rehabil 2016;97:974-82.

41. Hecksteden A, Faude O, Meyer T, et al. How to Construct, Conduct and Analyze an Exercise Training Study? Front Physiol 2018;9:1007.

42. Sterne JA, Davey Smith G. Sifting the evidence-what's wrong with significance tests? BMJ 2001;27:226-31. 